\title{
Laparoscopic Resection of Retroperitoneal Neural Tumors
}

\author{
Tetsuo Nozaki Tomonori Kato Akihiro Morii Hideki Fuse \\ Department of Urology, Graduate School of Medicine and Pharmaceutical Sciences for Research, University of Toyama, Toyama, Japan
}

\author{
Key Words \\ Laparoscopy • Retroperitoneal neural tumor • \\ Schwannoma • Ganglioneuroblastoma
}

\section{Abstract}

Purpose: Retroperitoneal neural tumor (RNT) is rarely excised laparoscopically, and the laparoscopic management of RNT remains controversial. We herein report 4 cases of laparoscopic excision of RNT that resulted in diverse clinical outcomes. Patients and Methods: Between August 2005 and January 2011, we performed laparoscopic excision of RNT in 4 patients. The mean tumor size was $4.5 \mathrm{~cm}$. The mean operative time was 297 minutes and the mean amount of blood loss was $55 \mathrm{ml}$. The surgeries were uneventful, with no operative complications or evidence of intra-abdominal bleeding. However, 2 patients required reoperation for delayed hemorrhage and urinoma formation, respectively. Results: The postoperative pathological diagnoses were schwannoma in 3 patients and ganglioneuroblastoma in 1 patient. All patients were well with no signs of peripheral neuropathy or radiculopathy, and CT and/or ${ }^{18} \mathrm{~F}-\mathrm{FDG}$ PET/CT performed during follow-up indicated no evidence of disease. Conclusions: Obtaining extensive preoperative knowledge of the source neural and vascular anatomy of the tumor is important for the surgical planning of laparoscopic resection of RNT. When a great deal of care is taken to divide the tumor and the source nerves and vital vessels, safe execution of RNT can be achieved for minimal postoperative mortality and morbidity.

Copyright $\odot 2013$ S. Karger AG, Basel

\section{Introduction}

Retroperitoneal neural tumor (RNT) usually becomes enlarged silently, until compression of the surrounding organs produces symptoms. RNTs have traditionally been excised using the standard open technique [1]. Due to their rarity, the use of the surgical modality of laparoscopic excision to treat RNTs is unusual; therefore, the significance of this operative strategy has not been completely evaluated. We herein report 4 cases of laparoscopic excision of RNT - 3 schwannomas and 1 ganglioneuroblastoma - that resulted in diverse clinical outcomes.

\section{Case Reports}

Between August 2005 and January 2011, 4 patients with RNT underwent laparoscopic excision. The patients' characteristics, operative data and clinical outcomes are summarized in table 1. The mean tumor size was $4.5 \mathrm{~cm}$. The mean operative time was 297 minutes and the mean amount of blood loss was $55 \mathrm{ml}$.

\section{Case 1}

A 42-year-old male was referred to our hospital for further evaluation of a right retroperitoneal tumor that had been discovered on abdominal computed tomography (CT) at the time of a routine health examination. The patient's medical history was unremarkable. Abdominal CT and magnetic resonance imaging (MRI) showed a $4.0 \times 3.5 \times 3.0 \mathrm{~cm}$, solid, well-circumscribed mass located posterior to the inferior vena cava and right renal vein. The imaging findings were compatible with a diagnosis of RNT, leiomyoma, rhabdomyoma or paraganglioma. The laborato-

\section{KARGER}

Fax +4161306 1234

E-Mail karger@karger.ch

www.karger.com
(C) 2013 S. Karger AG, Basel

1015-9770/13/0071-0040\$26.00/0

Accessible online at:

www.karger.com/cur
Tetsuo Nozaki

Graduate School of Medicine and Pharmaceutical Sciences for Research

Department of Urology, University of Toyama, 630 Sugitani

JP-9300194 Toyama (Japan)

E-Mail nozaki0921@yahoo.co.jp 
Table 1. Patients demographics

\begin{tabular}{|c|c|c|c|c|c|c|c|c|}
\hline Case & Sex & Age & Tumor size, $\mathrm{cm}$ & Tumor location & Operative time, $\min$ & $\mathrm{EBL}, \mathrm{ml}$ & Complication & Pathological diagnosis \\
\hline 1 & Male & 42 & 4.0 & $\begin{array}{l}\text { right periad- } \\
\text { renal }\end{array}$ & 203 & 10 & - & schwannoma \\
\hline 3 & Female & 59 & 3.0 & $\begin{array}{l}\text { left retroperi- } \\
\text { toneal, caudal } \\
\text { to the left renal } \\
\text { hilum }\end{array}$ & 172 & 10 & - & schwannoma \\
\hline 4 & Male & 59 & 1.8 & $\begin{array}{l}\text { left retroperito- } \\
\text { neal, adjacent } \\
\text { to the aorta, } \\
\text { psoas and spine }\end{array}$ & 325 & 150 & ureter injury & ganglioneuroblastomas \\
\hline
\end{tabular}

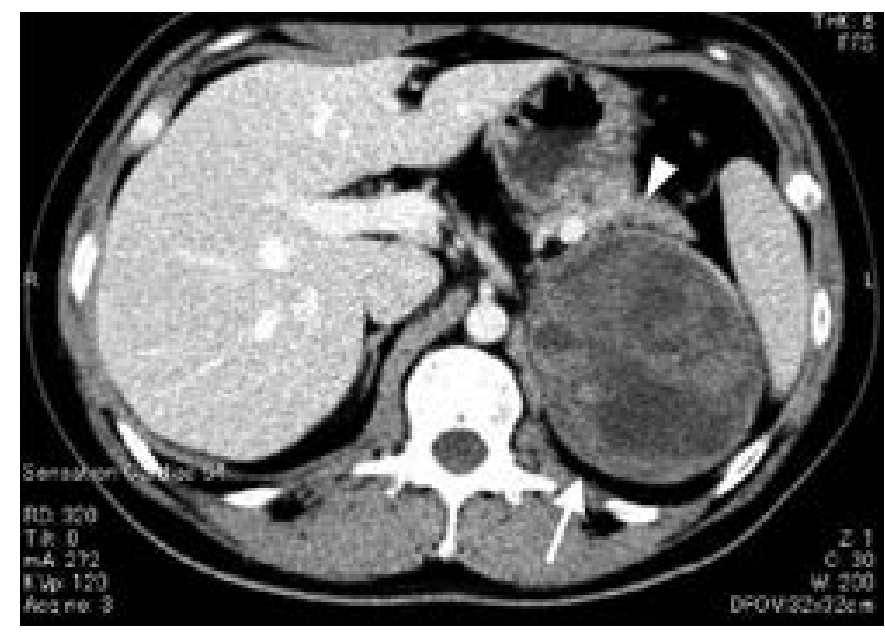

a

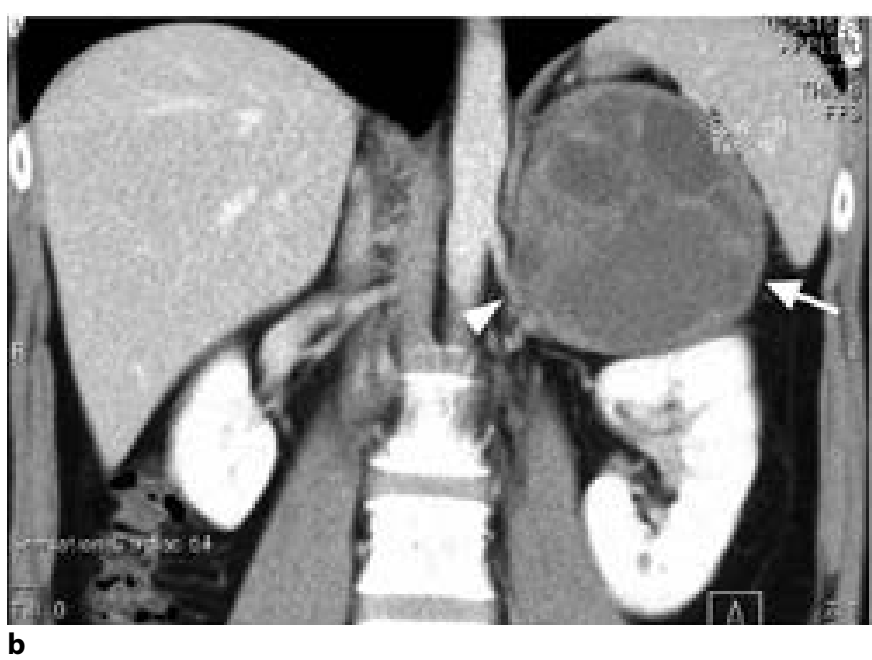

b

Fig. 1. Case 2: Left periadrenal RNT. aThe tumor (white arrow) compressed the pancreas (white arrowheads); b The tumor (white arrow) also compressed the left kidney and left renal artery (white arrowheads) in a caudal direction.

ry data and the levels of tumor markers were all within the normal limits, and a functional endocrine tumor test was negative. Operative excision was planned via laparoscopy, using an approach similar to that employed for right-sided adrenalectomy with the four-trocar technique. Adhesiolysis of the abdominal wall was performed, and the ascending colon was freed from the lateral gutter. After opening the Gerota's fascia, a soft elastic tumor was identified in the retroperitoneal space that appeared to be encapsulated and was easily dissected free from adjacent structures such as the inferior vena cava and right renal vein. We performed complete laparoscopic excision.

Laparoscopic Resection of

Retroperitoneal Neural Tumors
Case 2

A 36-year-old male presented to our hospital for further evaluation of a left retroperitoneal tumor that had been discovered on abdominal ultrasound at the time of a routine health examination. A physical examination was unremarkable. Abdominal enhanced CT and MRI revealed a $9.5 \times 9.0 \times 8.5 \mathrm{~cm}$, well-circumscribed left retroperitoneal mass (fig. 1). The laboratory data and the levels of tumor markers were all within the normal limits, and a functional endocrine tumor test was negative.

Laparoscopy was performed via an approach similar to that employed for left-sided adrenalectomy using the five-trocar tech- 

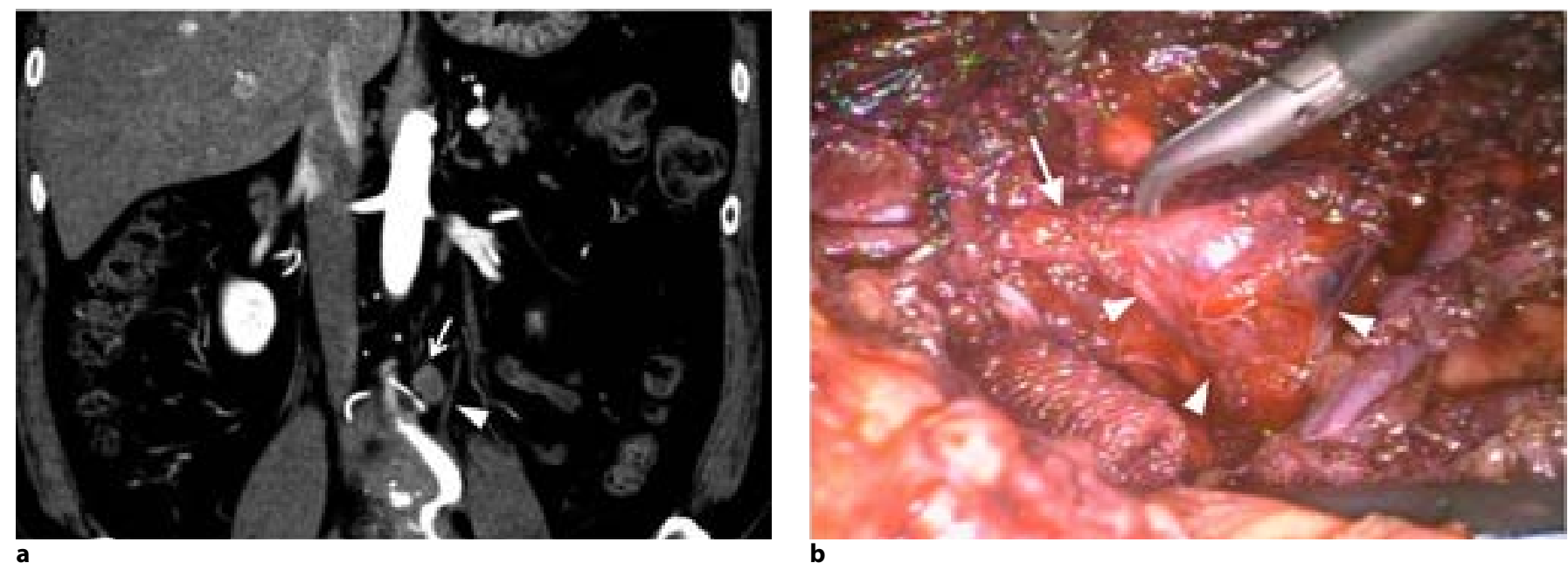

Fig. 2. Case 4: Left retroperitoneal recurrent RNT. a The tumor (arrow) was located adjacent to the aorta, psoas, spine and left ureter (arrowheads). b The source nerve (arrow) was surrounded by the RNT (arrowheads).

nique. Intraoperatively, a large elastic tumor was identified in the retroperitoneal space that was compressing the left adrenal gland medially. Although preoperative imaging findings did not indicate any evidence of local invasion, it was difficult to dissect the tissue between the tumor and the adjacent structures due to tight adhesion. The large RNT tumor and the left adrenal gland were completely removed en bloc using a harmonic scalpel. The operative time was 488 minutes and the estimated amount of blood loss was $50 \mathrm{ml}$. The surgery was uneventful with no operative complications or evidence of intra-abdominal bleeding. However, 5 hours after the operation, the patient developed hypotension and tachycardia, and laboratory tests showed a low hemoglobin level $(6.9 \mathrm{~g} / \mathrm{dl})$ (preoperative $12.5 \mathrm{~g} / \mathrm{dl})$. Because no obvious causes were evident clinically, the patient underwent urgent abdominal CT. CT scan demonstrated intraperitoneal fluid collection, suggesting delayed hemorrhage around the tumor bed. The patient's hemodynamic condition worsened and emergency reoperation was performed. After the removal of the intraperitoneal hematoma, laparotomic exploration showed active bleeding from the RNT feeding artery induced by dissection with a harmonic scalpel, which did not clip the artery. Further exploration within the abdomen showed no renal or splenic parenchymal injury, and hemostasis was completely achieved (the amount of intra-abdominal bleeding was $3,825 \mathrm{ml}$ ). The patient's postoperative course after the reoperation was uneventful, and he was discharged without further complaints.

Case 3

A 59-year-old female with severe hypertension, hypokalemia $(\mathrm{K}+1.77 \mathrm{mmol} / \mathrm{l})$ and the typical clinical features of primary aldosteronism was referred to our hospital. The plasma aldosterone concentration was elevated $(280 \mathrm{ng} / \mathrm{dl})$ and the plasma renin activity was suppressed $(0.3 \mathrm{ng} / \mathrm{ml} / \mathrm{h})$. Abdominal CT demonstrated a low-density left adrenal mass measuring $3.2 \mathrm{~cm}$ in diameter, and ${ }^{131} \mathrm{I}$-adrenal scintigraphy showed significant uptake by the left ad- renal gland. In addition, a left retroperitoneal tumor measuring 3.0 $\mathrm{cm}$ in diameter located just caudal to the left renal hilum was incidentally discovered. Simultaneous operative excision of the left adrenal gland and left retroperitoneal tumor was planned via laparoscopy. After laparoscopic left adrenalectomy was performed using the four-trocar technique, we opened the Gerota's fascia and a soft elastic tumor was identified between the left gonadal vein and left renal vein. The retroperitoneal tumor appeared to be encapsulated and was easily dissected free from adjacent structures using laparoscopic coagulating shears. We performed complete laparoscopic excision.

\section{Case 4}

A 59-year-old male was referred to our hospital for further evaluation of a left retroperitoneal tumor that had been discovered on abdominal CT. Eleven years previously, the patient had suffered from moderate pain in the left lumbosacral area.

Abdominal CT revealed an $8.0 \times 7.0 \mathrm{~cm}$ mass within the lower retroperitoneal pelvic space between the left external iliac artery and left internal iliac artery. The laboratory data and the levels of tumor markers were all within the normal limits, and a functional endocrine tumor test was negative. The retroperitoneal tumor was resected using conventional open surgery. The histopathological laboratory reported a completely resected ganglioneuroblastoma. After surgery, the patient underwent adjuvant irradiation (40 Gy) directed to the tumor bed. A follow-up abdominal CT scan performed after 11 years showed a 1.8 x $1.3 \mathrm{~cm}$ recurrent left retroperitoneal tumor adjacent to the aorta, psoas and spine (fig. 2a). It has been reported that despite surgery and radiation therapy or chemotherapy, the prognosis of adult onset ganglioneuroblastoma is not favorable. In order to avoid local recurrence and distant metastasis, we consider total resection with a wide surgical margin to be the best treatment for retroperitoneal recurrent ganglioneuroblastoma tumors. Preoperatively, we consulted the orthopedics department regarding possible postoperative neurological deficits 
following en bloc tumor resection with neural compromise. A preoperative evaluation including an MRI scan suggested that the tumor could be resected en bloc with neural compromise without after effects. After informing the patient, we planned laparoscopic resection of the retroperitoneal recurrent ganglioneuroblastoma tumor. An incision was made along the line of Told. The descending colon, kidney and spleen were extensively reflected medially, and the plane anterior to the psoas muscle was completely developed. A hard elastic tumor originating from the lower lumbar-sacral plexus was identified on the left para-aortic side between the lumbar vertebrae and left psoas muscle. The tumor appeared to be adhered to the left ureter. The tumor was carefully dissected from the left ureter using laparoscopic coagulating shears. For complete resection, performing slightly traumatic dissection was necessary due to the presence of dense adhesions around the tumor and the effects of prior laparotomy and radiation therapy. As a result of difficulty in mobilizing the mass from the nerve root, we decided to resect the tumor en bloc with the nerve root because the tumor pathology indicated malignancy (fig. 2b). After the tumor was isolated circumferentially from the surrounding tissue, hemostasis was achieved with bipolar electrocautery. The excised specimen was placed into an endobag and removed. The patient has been making satisfactory progress since the operation, exhibiting full mobility. However, he developed left lumbosacral pain 5 days after undergoing surgery. An abdominal CT scan indicated the presence of a retroperitoneal urinoma. A percutaneous drain was placed, and a left nephrectomy procedure was required 12 days after the laparoscopic surgery to control an infection caused by the urinoma. The patient's condition gradually improved postoperatively. The final histopathological report indicated a ganglioneuroblastoma with a malignant component. The patient remained well with no signs of peripheral neuropathy or radiculopathy and was discharged from the hospital 20 days later without receiving adjuvant chemotherapy or irradiation. Follow-up abdominopelvic CT and ${ }^{18} \mathrm{~F}$-FDG PET/CT performed at the 12-month postoperative visit indicated no evidence of disease.

\section{Discussion}

Schwannomas are neurogenic tumors that arise from Schwann cells in peripheral nerves. Das Gupta et al. [2] reported that schwannomas primarily occur in the cephalocervical region $(44.8 \%)$, limbs $(32.6 \%)$ and occasionally the retroperitoneal space $(0.7 \%)$. In cases of retroperitoneal schwannomas, since the patients are often asymptomatic or have only vague abdominal pain, the schwannomas may be identified incidentally when other complaints are investigated or may grow very large before causing overt symptoms. Establishing a preoperative diagnosis is not easy, although conducting radiographic imaging is helpful for therapeutic planning, as it provides information about tumor size, tumor location and possible invasion of other structures. The first-line treatment for schwannomas is surgical resection, and the principal procedure is total resection, including remov-

Laparoscopic Resection of

Retroperitoneal Neural Tumors al of the capsule. Incomplete excision may lead to local recurrence (in approximately $10-20 \%$ of cases) [3, 4]. Laparoscopic surgery, which enables the surgeon to obtain detailed observations with minimal invasiveness and magnified vision, has become a viable option for this procedure. Recently, many researchers have reported that it is feasible to treat smaller schwannomas under a laparoscopic approach [5-10]. However, the management of larger schwannomas $(>5 \mathrm{~cm})$ remains controversial. It has been reported that larger schwannomas tend to prolong the length of hospital stay (3-18 postoperative days) after laparoscopic resection, while shorter hospital stays (approximately 5 postoperative days) were noted in a recent report of larger retroperitoneal schwannomas treated with the open approach [11]. Moreover, if the mass is strongly connected to essential organs, iatrogenic complications (neural deficits, vascular lesions) may occur in $10 \%$ of cases. Bleeding may be also anticipated when the tumor capsule is adherent to the venous plexus and a high volume of blood loss might be encountered $[11,13]$. Surgeons should bear this in mind, because as schwannomas grow larger, performing surgery becomes more difficult since the source nerve is surrounded by the mass, which also adheres in tight connection to adjacent vessels. Therefore, a great deal of care must be taken to divide the tumor and the source nerves and vital vessels. If complete resection cannot be performed safely, the operation should not be continued laparoscopically. In patients with local invasion, conversion to an open procedure should be an early decision, made prior to tumor morcellation or fracture of the tumor capsule.

Ganglioneuroblastoma is uncommon malignant tumor that develops in the sympathetic nervous system and adrenal glands. This neoplasm usually occurs in children and is extremely rare in adults. To our knowledge, only 48 cases of adult onset ganglioneuroblastoma have been reported in the literature [14-20]. Among these cases, 12 involved the adrenal glands (25.0\%), which are the most frequent site. The second most frequent site of occurrence is the mediastinal space ( 8 cases, $16.7 \%$ ), followed by the retroperitoneal space (6 cases, $12.5 \%$ ). Other sites include the neck, brain, nasal fossa and ganglion, reflecting that these tumors may arise in any location along the sympathetic nervous system [20]. On diagnosis, most cases of adult onset ganglioneuroblastoma are at an advanced stage. Therefore, only a few patients may be good candidates for a curative surgical approach. Among cases of adult ganglioneuroblastoma with distant metastasis, the patients died within a mean period of 10.7 months (range from 2 weeks to 3 years). 
All patients treated curatively have exhibited favorable prognoses, and none have died of recurrence (mean follow-up period 18.8 months) [20]. In our case, because the prognosis was not favorable despite the administration of radiation therapy and chemotherapy, we considered total resection with a wide surgical margin to be the best treatment for recurrent retroperitoneal ganglioneuroblastoma tumors. The present case was unique in that the tumor was removed laparoscopically. There has been only one report regarding the laparoscopic removal of a ganglioneuroblastoma tumor in an adult. Koike et al. [14] reported their experience with laparoscopic resection of an adrenal ganglioneuroblastoma. They concluded that the precise indications for laparoscopic resection of an abdominal ganglioneuroblastoma provide a better prognosis and a good postoperative quality of life for the patient. In our case, although complete removal of the tumor was accomplished, a retroperitoneal urinoma developed due to the relatively traumatic dissection performed during ureterolysis. Although preoperative imaging did not show any evidence of local invasion, the presence of dense adhesions around the tumor was indicated.
On reflection, proximity of the lesion to the ureter may require preoperative ureteral catheterization. Moreover, the source nerve was surrounded by the mass, and we decided to resect the tumor en bloc with the source nerve root because the tumor pathology indicated malignancy and complete resection constitutes the only possibility of a cure. Fortunately, the patient has remained well with no signs of peripheral neuropathy or radiculopathy, and no recurrence has been noted for 1 year to date, although further careful follow-up is warranted.

In conclusion, obtaining extensive preoperative knowledge of the source neural anatomy and vascular anatomy of the tumor is important for the surgical planning of laparoscopic resection of RNT, which differs in each individual situation. Very close communication between the surgical and orthopedics teams is essential. Such teamwork and preoperative planning should have the goal of achieving safe execution of RNT for minimal postoperative mortality and morbidity. When accurate and appropriate preoperative plans are made, the use of laparoscopic surgical techniques to treat retroperitoneal RNT is safe and encouraged.

\section{References}

-1 Shindo S, Motohashi S, Katsu M, Kaga S, Inoue $\mathrm{H}$, Matsumoto $\mathrm{M}$, Kono K, Fujii $\mathrm{H}$, Takeda M: Treatment of abdominal malignancy invading the vena cava: a report of seven cases. Surg Today 2007;37:685-689.

-2 Das Gupta TK, Brasfield RD, Strong EW, Hajdu SI: Benign solitary Schwannomas (neurilemomas). Cancer 1969;24:355-366.

$\checkmark 3$ Daneshmand S, Youssefzadeh D, Chamie K, Boswell W, Wu N, Stein JP, Stein JP, Boyd S, Skinner DG: Benign retroperitoneal schwannoma: a case series and review of the literature. Urology 2003;62:993-997.

4 Tortorelli AP, Papa V, Rosa F, Pacelli F, Doglietto GB: Image of the month-retroperitoneal schwannoma. Arch Surg 2006;141: 1259-1261.

-5 Ningshu L, Min Y, Xieqiao Y, Yuanqing Y, Xiaoqiang M, Rubing L: Laparoscopic management of obturator nerve schwannomas: experiences with 6 cases and review of the literature. Surg Laparosc Endosc Percutan Tech 2012;22:143-147.

-6 Lacarriere E, Le Long E, Caremel R, Grise P: Removal of pelvic schwannoma using a retroperitoneoscopic and open double approach: description of an effective novel technique. J Laparoendosc Adv Surg Tech A 2012;22: 269-272.
7 Seo IY, Boldbaatr Y, Choi KH: Laparoscopic resection of ancient schwannoma embedded in the psoas muscle. Surg Laparosc Endosc Percutan Tech 2011;21:e336-338.

$>8$ Pazouki A, Khalaj A, Shapoori P, Vaziri M, Najafi L: Laparoscopic resection of a retroperitoneal schwannoma. Surg Laparosc Endosc Percutan Tech 2011;21:e326-328.

$>9$ Gorgun M, Sezer TO, Kirdok O: Laparoscopic resection of retroperitoneal schwannoma near the inferior vena. Ann Vasc Surg 2010; 24:551.e1-4.

10 Sasaki A, Suto T, Nitta H, Shimooki O, Obuchi T, Wakabayashi G: Laparoscopic excision of retroperitoneal tumors: report of three cases. Surg Today 2010;40:176-180.

$\checkmark 11$ Yoshino T, Yoneda K: Laparoscopic resection of a retroperitoneal ancient schwannoma: a case report and review of the literature. Anticancer Res 2008;28:2889-2891.

$\checkmark 12$ Melvin WS: Laparoscopic resection of a pelvic schwannoma. Surg Laparosc Endosc 1996;6:489-491.

13 Descazeaud A, Coggia M, Bourriez A, Goeau-Brissonniere O: Laparoscopic resection of a retroperitoneal schwannoma. Surg Endosc 2003; 17:520.

$>14$ Koike K, Iihara M, Kanbe M, Omi Y, Aiba M, Obara T: Adult-type ganglioneuroblasto$\mathrm{ma}$ in the adrenal gland treated by a laparoscopic resection: report of a case. Surg Today 2003:33:785-790.
15 Slapa RZ, Jakubowski W, Kasperlik-Zaluska AA, Szopinski K, Debski R, Samsel M, Polanski J, Borowicz K: Adrenal ganglioneuroblastoma in pregnant woman: diagnosis with three-dimensional ultrasound. Eur Radiol 2002:12:S121-126.

16 Nakazato Y, Hosaka N: A 32-year-old man with left temporal lobe tumor. Neuropathology 2004;24:261-262.

17 Gunlusoy B, Arslan M, Selek E, Sural S, Ayder AR: A case report: adrenal ganglioneuroblastoma in a 59-year old man. Int Urol Nephrol 2004;36:481-483.

18 Kurt E, Filiz G, Vuruskan H, Kurt M, Evrensel T: Life-threatening complications of an extremely rare tumor of the kidney: adulttype primary renal ganglioneuroblastoma. South Med J 2007;100:852-853.

$>19$ Nishihara H, Ozaki Y, Ito T, Yoshinaga T, Tabu K, Tanino M, Nagashima K, Tanaka $\mathrm{S}$ : A case of cerebral ganglioneuronal tumor in the parietal lobe of an adult. Brain Tumor Pathol 2008;25:45-49.

20 Mizuno S, Iida T, Fujita S: Adult-onset adrenal ganglioneuroblastoma - bone metastasis two years after surgery: report of a case. Surg Today 2010;40:482-486. 